admission to a mental hospital, many of them in the first week. Two possible explanations may be suggested. The first is that selection of patients for admission was ill-judged ; the second is that the staff and facilities of a mental hospital are inadequate to treat gross organic illness. Or was it a matter of Hobson's choice, getting the patient into any bed available ? At present the ills of old age are not adequately provided for. Old people often cannot be sorted satisfactorily by whether their illness is mental or physical. If it is evident that the family or the community can no longer cope, the old people should surely be cared for in hospital with no thought of whether they are "geriatric" or " psychiatric."

\section{Familial Mediterranean Fever}

Familial Mediterranean fever occurs most frequently in Sephardic Jews, occasionally in Arabs and other inhabitants of the Mediterranean littoral, and very rarely in Gentiles in Western Europe. ${ }^{1}$ The condition is inherited as an autosomal recessive, and is more common in males than females. Though never manifest at birth, familial Mediterranean fever may present as early as the end of the first year of life, but its maximum incidence is between the ages of 10 and 20 years. There are two types of illness. The first, and common type, consists of attacks of fever and pain ; the second is characterized by the insidious onset of amyloidosis.

In the first type of illness there are short episodes of fever. These occur at unpredictable intervals and may be accompanied by pain in the abdomen, joints, and chest, in that order of frequency. Attacks of abdominal pain may suggest an acute abdominal catastrophe, but characteristically the attack subsides in 6 to 12 hours and the patient is then well. If laparotomy is done for, say, suspected appendicitis, the peritoneum appears hyperaemic and a small amount of cloudy exudate is seen ; occasionally adhesions may occur and may cause intestinal obstruction. Pain in the chest is pleuritic in type and is usually accompanied by a small pleural effusion, but again these resolve rapidly. By contrast, involvement of the joints may be prolonged. Usually only one joint is affected at a time, and this shows all the clinical features of an acute pyogenic infection, though the effusion is found to be sterile. Complete return of the joint to normal is the rule even when definite osteoporosis in the subarticular region has been present. Another characteristic of familial Mediterranean fever is an erysipelas-like erythema, which occurs on the legs. Occasionally short attacks of low-grade fever occur as the only manifestation.

In the second type of illness the first sign of the characteristic amyloidosis is transient proteinuria, which progresses to the nephrotic syndrome, uraemia, and death. A similar pattern of amyloidosis also supervenes in the first type of the disease. In both types of illness amyloidosis affects the kidney almost exclusively; the spleen is involved only rarely and the liver never. Though biochemical studies of the amyloid have been unhelpful, investigations using polarization and electronmicroscopy have shown that amyloid is probably formed by cells of the fibroblast series. Once symptoms

\footnotetext{
' Sohar, E., Gafni, J., Pras, M., and Heller, H., Amer. \%. Med., 1967, 43, 227.

- Bondy, P. K., Cohn, G. L., and Gregory, P. B., Medicine (Baltimore), the Year 1964, Part III, Commentary, 1967.' H.M.S.O.
}

from amyloid disease have developed the prognosis is poor, and survival for more than a few years is unlikely.

The differential diagnosis of familial Mediterranean fever includes a variety of disease associated with transient attacks of fever and pain. Disorders of collagen and the reticuloses may be simulated. A rare form of fever associated with increased circulating aetiocholanolone may be mentioned, as it can easily be confused. ${ }^{2}$ This type, however, does not occur in Jewish people, but affects northern races; it usually starts in childhood, and amyloidosis is not a sequel ; and it responds well to corticosteroids. This response to therapy is in marked contrast with familial Mediterranean fever, in which there is little that can be done either symptomatically or to delay the onset of amyloid formation.

\section{Therapeutic Misadventure}

The latest Commentary volume issued by the Registrar General for England and Wales ${ }^{1}$ is again a useful reminder of some of the uncommon, but recurring, causes of deaths. A group deserving the special attention of the medical profession comprises the deaths due to therapeutic misadventure, periodically analysed at the General Register Office. In 1965, the year now reviewed, 235 deaths were in this category, of which 46 were due to radiotherapy, 10 to blood transfusion, and 9 to anaesthesia. In addition to a few more due to $x$-ray contrast media, electric convulsion treatment, and miscellaneous causes, about 150 were the result of reactions to medicaments of various kinds. The largest single group was 50 deaths from corticosteroid therapy, and this was followed by a group of 20 attributed to phenylbutazone or oxyphenbutazone.

The total of 235 deaths in this category as well as the figures for corticosteroids and the butazones are sharp reverses to trends that had persisted for several years. In $1964^{2}$ the total (103) had fallen for the third successive year and was the lowest for some years, so that the Registrar General ${ }^{3}$ was moved to welcome the trend "with restrained satisfaction." Unfortunately the 1965 figures upset this interpretation, and though, as might be expected, remedies vary over the years in the extent to which they cause death from misadventure, several groups of them still stand out for the risk that must be weighed against the undoubted benefit they offer. Apart from the corticosteroids and butazones, other substances shown as causes of deaths from therapeutic misadventure (with numbers of deaths) are as follows : anticoagulants (18), chloramphenicol (5), phenacetin (5), aspirin and salicylate (4), penicillin (3), and tetracyclines (3).

Five deaths in 1965 were associated with vaccination against smallpox and four with immunization against other diseases. In the latter group was a man aged 22 reported to have died from anaphylactic shock after having oral poliomyelitis vaccine. Of 215 deaths caused by accidental overdose of a drug (including self-administered remedies), 183 $(85 \%)$ were due to barbiturates and other hypnotic drugs, $141(66 \%)$ being due to barbiturates alone.

\footnotetext{
1 The Registrar General's Statistical Review of England and Wales for the Year 1965, Part III, Commentary, 1968. H.M.S.O.

2 See Brit. med. Э., 1967, 3, 66.

- The Registrar General's Statistical Review of England and Wales for $1965,44,249$.
} 